\title{
In Search of Surface-Induced Crystal Structures: The Case of Tyrian Purple
}

\author{
Lorenzo Pandolfi, Arianna Rivalta, Tommaso Salzillo,* Andrea Giunchi, Simone D’Agostino, \\ Raffaele G. Della Valle, Aldo Brillante, and Elisabetta Venuti*
}

Cite This: J. Phys. Chem. C 2020, 124, 17702-17710

Read Online

ACCESS I

Llll Metrics \& More

Article Recommendations

Supporting Information

ABSTRACT: Recently, structural studies in the thin-film phase of $6,6^{\prime}$-dibromoindigo, the main component of the Tyrian purple dye, revealed the presence of surface-induced structures. This finding is important, as the system is a good candidate for applications in organic electronics, and knowledge of the film structure is needed to explain the physical properties of the conducting layer. In this work, combining the results of low-frequency Raman spectroscopy, X-ray diffraction, and accurate density-functional theory (DFT) calculations of the vibrational properties of the proposed surfaceinduced structures, we suggest that a newly detected $6,6^{\prime}$ dibromoindigo bulk crystal form is structurally closely related to the surface-induced modifications. Thus, 6,6' ${ }^{\prime}$-dibromoindigo behavior shows similarities and differences at the same time, with respect to other investigated systems.

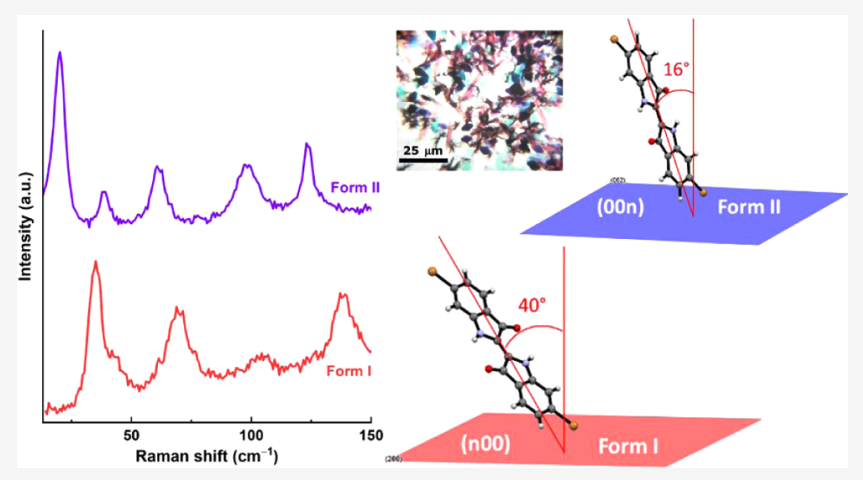

\section{INTRODUCTION}

Belonging to the large class of substances named indigoids after the parent molecule, the compound 6,6'-dibromoindigo (Figure 1) has been identified long since as the main

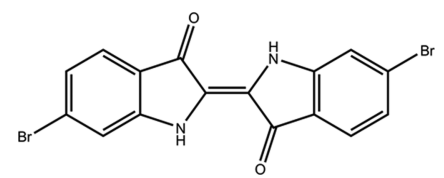

Figure 1. Molecular structure of 6,6' $6^{\prime}$ dibromoindigo (6,6'-dibromo$1 H, 1^{\prime} H$ - $\left[2,2^{\prime}\right]$ biindolylidene-3,3'-dione).

component of the precious pigment Tyrian purple. ${ }^{1-3}$ In the past years, a few reports have highlighted the ambipolar semiconducting properties of 6,6'-dibromoindigo in thin-film organic field-effect transistors (OFETs), a characteristic in common with other systems of the class, with this compound in fact outperforming indigo., ${ }^{4,5}$ In turn, its potential in applications recently found the theoretical support of a densityfunctional theory (DFT) study on indigoid-based singleelectron transistor nanodevices. ${ }^{6}$

While it has been noted that indigoid's best OFET performances can be observed on apolar dielectrics, a behavior ascribed to a standing-up molecular orientation on these substrates, ${ }^{7}$ efforts to clarify structural aspects of the growth at the dielectric interface have been made. As different crystal modifications might be responsible for specific interface orientations, the detailed structural investigation of the films employed in organic electronics is part of the fundamental research needed to explain the physical properties of the conducting layer.

Most investigations have involved indigo, ${ }^{8-10}$ the mobility of which on hydrocarbon substrates has been attributed to a surface-induced crystal structure, nucleating via a mechanism of epitaxial growth. ${ }^{8}$ However, the structural parameters of such a modification are in fact unknown. Both on polar substrates such as glass or silicon oxide and on the slightly polar Tetoron, ${ }^{11,12}$ we have proved that only one of the two indigo bulk structures, namely, the B form, can be found, independent of the method of growth and unexpectedly considering the previously reported instability of this polymorph. ${ }^{13-15}$ Surface phase selectivity has been demonstrated also for the synthetic dye thioindigo, with the metastable polymorph $\alpha$ growing preferentially on silicon oxide surfaces, at the expense of the thermodynamically stable form $\beta .^{16}$

Various slightly different thin-film phases of 6,6'-dibromoindigo were identified on a number of substrates, and one of the

Received: June 8, 2020

Revised: July 21, 2020

Published: July 21, 2020 
structures detected on silicon oxide was solved in a combined theoretical and experimental approach, ${ }^{17,18}$ which used grazing incidence X-ray diffraction (GIXRD) and molecular dynamics (MD) simulations. Therefore, two kinds of structures appeared to exist for this system: one for the already known bulk phase, ${ }^{2,3}$ indeed very similar to either of the monoclinic indigo polymorphs, ${ }^{13,14}$ and the second one triclinic, belonging to surface-mediated structures. In this respect, Tyrian purple constitutes an interesting case, as it seemed to display a genuine surface-induced structure, being, at the same time, one of the indigoids for which polymorphism in the bulk state was not reported. A relevant feature of the surface-mediated structures is that in these the molecules are organized with the long molecular axis forming a small angle with the normal to the contact plane, i.e., in the geometry assumed to be optimal for charge transport.

The work presented here is part of an in-depth investigation aimed to probe the relationship between crystal structures and physical properties of organic dyes ${ }^{11,12,16,19}$ and focuses on 6,6'-dibromoindigo. As demonstrated previously, ${ }^{11,12,16,19}$ an efficient association of XRD techniques and low-frequency Raman spectroscopy can be exploited for the characterization of polymorphs. In addition, highly accurate DFT calculations ${ }^{20,21}$ allow for the reproduction in frequency and intensity of the low-frequency vibrational spectra of a crystal modification, just starting from a tentative structure, much in the same way as the X-ray analysis is conducted. This implies that a polymorph identification can be made in a straightforward way, by comparing spectroscopic measurements and calculations.

The investigation of the solid state of $6,6^{\prime}$-dibromoindigo has been conducted on both single crystals and samples obtained by vapor methods or solution deposition on glass. The latter process yields a quite homogeneous crystalline deposit, whose growth is influenced by the presence of the substrate. The investigation has led to the identification of a new bulk form, whose Raman spectrum and diffraction pattern match quite closely those simulated for the surface-induced structures of the literature. Also, in common with such structures is the phase transition to the bulk form on heating. This suggests that the surface-mediated structures can be associated with a crystal modification, which is already present in the polymorph energy landscape of the compound bulk forms.

\section{EXPERIMENTAL METHODS}

2.1. Crystal Growth. 6,6'-Dibromoindigo (6,6'-dibromo$1 \mathrm{H}, 1^{\prime} \mathrm{H}$-[2,2'] biindolylidene-3,3'-dione, Endotherm $\mathrm{GmbH}$, purity $>95 \%$ ) was purified by sublimation at $200{ }^{\circ} \mathrm{C}$, in a vacuum of $\mathrm{N}_{2}$ atmosphere. The purified product is a crystalline powder, with a varying polymorph composition (see the Results and Discussion section).

Single crystals of the known bulk form were obtained by the physical vapor transport (PVT) method, in the apparatus described elsewhere. ${ }^{11}$ Evaporation took place at $250^{\circ} \mathrm{C}$, and the vapor diffused under a temperature gradient of $\approx 8{ }^{\circ} \mathrm{C} / \mathrm{cm}$ to a cold end set at $150{ }^{\circ} \mathrm{C}$. The growth process took two weeks and yielded crystal platelets.

Growth by solution deposition was made by the dropcasting technique: $1 \mathrm{mg}$ of sublimated powder was dissolved in $10 \mathrm{~mL}$ of dimethyl sulfoxide (DMSO) (Aldrich), with a concentration of $\approx 2 \times 10^{-4} \mathrm{M}$, heated at $150{ }^{\circ} \mathrm{C}$, filtered $(0.2$ $\mu \mathrm{m}$ filter) and deposited, dropwise, on a clean glass substrate heated at $150{ }^{\circ} \mathrm{C}$. After solvent evaporation, a deposit of microcrystals was obtained, in which two different characteristic shapes of plates and needles were clearly evidenced. Under a polarizer microscope, the platelets appeared blue, while the needle-shaped crystals had a pale purple color.

2.2. Raman Measurements. Raman spectra of all of the crystalline samples in the lattice phonon region (10-150 $\mathrm{cm}^{-1}$ ) were collected with a Horiba Jobin Yvon T64000 triple monochromator spectrometer interfaced with the optical stage of an Olympus BX40 microscope. The excitation wavelength was from the $647.1 \mathrm{~nm}$ line of a $\mathrm{Kr}^{+}$laser, the same used for the parent molecule indigo, ${ }^{11}$ with the exception of the commercial product, which required the $514.5 \mathrm{~nm}$ excitation from an $\mathrm{Ar}^{+}$laser, to more efficiently reduce the background emission of its impurities. A half-wave plate was used to rotate the polarization of the incident light, while a wire grid polarizer selected the polarization of the scattering. Extinction directions allowed us to orient the crystal specimens for measurements in polarized light.

For a better analysis of the high-wavenumber region (100$\left.2000 \mathrm{~cm}^{-1}\right)$, we employed an inVia Renishaw single monochromator spectrometer equipped with a suitable edge filter and coupled to a Leica DM LM microscope. For these measurements, the excitation wavelength was always from the $514.5 \mathrm{~nm}$ line of an $\mathrm{Ar}^{+}$laser, whose output power of $25 \mathrm{~mW}$ was reduced to $10 \%$ to avoid sample damage.

2.3. X-ray Diffraction. Single-crystal data for $6,6^{\prime}$ dibromoindigo grown from PVT were collected at RT on an Oxford X'Calibur S CCD diffractometer equipped with a graphite monochromator (Mo K $\alpha$ radiation, $\lambda=0.71073 \AA$ ) and identified as Form I. Data collection and refinement details are listed in Table S1. All non-hydrogen atoms were refined anisotropically. $\mathrm{H}_{\mathrm{CH}}$ atoms were added in calculated positions and refined riding on their respective carbon atoms; $\mathrm{H}_{\mathrm{NH}}$ atoms were directly located on the heteroatoms. SHELX $97^{22}$ was used for structure solution and refinement on $\mathrm{F}^{2}$. The program Mercury ${ }^{23,24}$ was used to calculate intermolecular interactions and for molecular graphics.

For phase identification purposes, X-ray powder diffractograms in the $2 \theta$ range $5-40^{\circ}$ (step size, $0.02^{\circ}$; time/step, $20 \mathrm{~s}$; $0.04 \mathrm{rad}$ soller; $40 \mathrm{~mA} \times 40 \mathrm{kV}$ ) were collected on a Panalytical X'Pert PRO automated diffractometer equipped with an X'Celerator detector and in Bragg-Brentano geometry, using $\mathrm{Cu} \mathrm{K} \alpha$ radiation without a monochromator. The same settings were used to collect data on polycrystalline films grown on a glass substrate. The program Mercury ${ }^{23,24}$ was used for simulation of X-ray powder patterns on the basis of single-crystal data. Chemical and structural identities between the bulk material and single crystal were verified by comparing experimental and simulated powder diffraction patterns; see the Supporting Information. For structure refinement purposes, X-ray powder diffractograms in the $2 \theta$ range $5-70^{\circ}$ (step size, $0.026^{\circ}$; time/step, $199 \mathrm{~s} ; 0.02 \mathrm{rad}$ soller; $40 \mathrm{~mA} \times 40 \mathrm{kV}$ ) were collected on a Panalytical X'Pert PRO automated diffractometer operated in transmission mode (capillary spinner) and equipped with a pixel detector. Powder diffraction data were refined by the Rietveld method with software TOPAS 4.1. ${ }^{25}$ A shifted Chebyshev function with 7 parameters and a Pseudo-Voigt function (TCHZ type) were used to fit the background and peak shape, respectively. A spherical harmonics model was used to describe the preferred orientation; see the Supporting Information for difference pattern plots and associated figures of merit (FOM). 
Crystal data can be obtained free of charge via www.ccdc. cam.ac.uk/conts/retrieving.html (or from the Cambridge Crystallographic Data Center, 12 Union Road, Cambridge CB21EZ, UK; fax: (+44)1223-336-033; or e-mail: deposit@ ccdc.cam.ac.uk). CCDC numbers are 2006175 and 2006176.

2.4. Computational Methods. DFT simulations of $6,6^{\prime}$ dibromoindigo bulk structure Form $\mathrm{I}^{2,3}$ and surface-induced modifications (SIPs) ${ }^{17,26}$ used the VASP (Vienna Ab initio Simulation Package) code, ${ }^{27-30}$ with the Perdew-BurkeErnzerhof (PBE) exchange correlation functional ${ }^{31}$ and with the projected-augmented wave (PAW) pseudopotentials PAW_PBE $\mathrm{H}$ and PAW_PBE C. ${ }^{32,33}$ Dispersive interactions were taken into account by the D3-BJ pairwise correction (DFT-D3). ${ }^{34}$ Energy convergence was achieved for the various structures as follows: a plane wave cutoff of $800 \mathrm{eV}$ proved to be adequate in combination with $2 \times 5 \times 2$ Monkhorst-Pack $k$-point sampling for Form I and $6 \times 4 \times 2$ sampling for the SIP modifications. An increase in the cutoff energy from 800 to $1200 \mathrm{eV}$ caused energy variations slightly larger than $1 \mathrm{meV} /$ atom, while the denser $k$-point samplings of $4 \times 10 \times 4$ for Form I and $10 \times 7 \times 3$ for the SIPs gave energy changes around $0.1 \mathrm{meV} /$ atom. Less dense $k$-point samplings actually yield the same accuracy. Atomic coordinates (or atomic coordinates and cell parameters) were fully relaxed, halting when residual forces fell below $1 \mathrm{meV} / \AA$, using the GADGET package. $^{35}$ For both energy cutoffs and $k$-point sampling, energy differences between polymorphs converge faster than absolute energies to within $0.04 \mathrm{meV} /$ atom.

Calculated and experimental crystallographic structures were compared by the distance comparison method ${ }^{36}$ in which all of the interatomic distances between a reference molecule and $N$ neighboring molecules in a spherical coordination shell are listed, and pairs of structures are then compared by means of the root-mean-square deviation, $\mathrm{RMSD}_{N}$, between their lists of distances. The method was also used to compare the various SIP minima to probe whether they can be associated with the same cluster of structures.

Vibrational modes, restricted to the $\Gamma$ point, were computed at the experimentally determined unit cell volumes through the force constants obtained with PHONOPY software ${ }^{37}$ in combination with VASP. Polarizability tensors $\alpha$ for each crystal mode were obtained using the Python program vasp_raman.py, ${ }^{38}$ which uses the VASP code as the back end. Raman intensities were finally adjusted by considering the excitation wavelength and temperature dependence. ${ }^{39}$ Polarized Raman spectra were calculated as described in detail in ref 16.

\section{RESULTS AND DISCUSSION}

\subsection{Characterization of Single-Crystal and Powder} Samples. The $Z=2$ monoclinic crystal structure determined in this work for the PVT-grown samples, $P 21 / c$, with cell parameters (in $\AA$ and ${ }^{\circ}$ ) $a=12.5512(7), b=4.8510(3), c=$ 11.5644(7), $\beta=103.935(6)$, and $V / Z=343.28(7) \AA^{3}$, corresponds to the only one reported in the earlier literature ${ }^{2,3}$ and is named from here on Form I. Crystal data and refinement details can be found in Table S1. The molecular arrangement is similar to that of the indigo polymorphs, driven by the formation of strong intermolecular hydrogen and halogen bonds and $\pi-\pi$ stacking, as shown in Figure 2 . When molecular geometries of indigo and 6,6'-dibromoindigo are compared, it is evident how bromination does not substantially

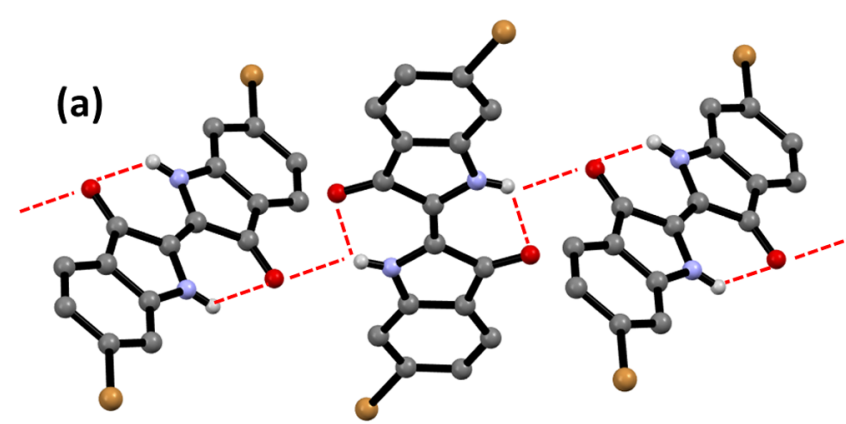

(b)

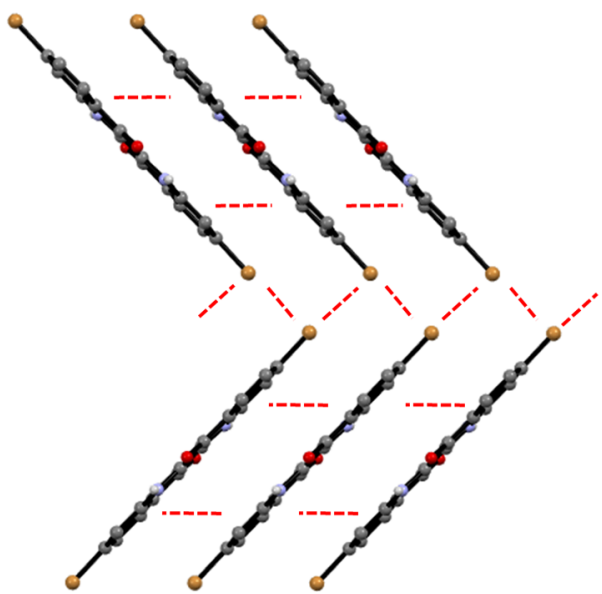

Figure 2. Intermolecular interactions detected in crystalline $6,6^{\prime}$ dibromoindigo Form I: (a) hydrogen bonds $\left[\mathrm{dN}_{\mathrm{NH}} \cdots \mathrm{O}_{\mathrm{CO}}\right.$, intermolecular $=2.938(4) \AA$ and intramolecular $=2.916(4) \AA]$; (b) $\pi$-stacking $[3.4 \AA]$ and halogen bonds $\left[\mathrm{d}_{\mathrm{Br} \cdots \mathrm{Br}}=3.749(8) \AA\right.$, angle $\left.\mathrm{Br}_{\mathrm{Br}-\mathrm{Br}}=86.34(1)^{\circ}\right] . \mathrm{H}_{\mathrm{CH}}$ atoms are omitted for clarity.

modify the parent molecule ring system and the interaction pattern.

For this polymorph, the DFT D3-computed versus experimental $\mathrm{RMSD}_{N}$ distance at the minimum energy structure was found to be $0.10 \AA$, which confirms the excellent reliability of the calculation results (see Table S2 for further details).

Figure 3 shows the Raman spectrum of a single crystal of Form I obtained by the PVT method and measured at an excitation wavelength of $514.5 \mathrm{~nm}$.

The wavenumber range $\left(10-150 \mathrm{~cm}^{-1}\right)$ reported in the figure allows for the detection of both the lattice phonon vibrations, which represent the fingerprint of the crystal structure, and intramolecular low-energy modes. A detailed vibrational assignment of the spectrum of Form I over this range can be made by means of experiments and calculations in polarized light and will be given below. In Figure 3, the spectrum of the single crystal is compared with those of the microcrystalline powders of both the commercial product and the sample purified by sublimation.

Some of the observed spectral differences, like the merging of some bands into a broad envelope in the commercial product, are expected, as the comparison is made between a single crystal and powderlike specimens. In the powders, however, the appearance of a new vibrational pattern is clearly detectable, even in the presence of some of the strongest bands of Form I. Namely, the peak at $35 \mathrm{~cm}^{-1}$ with its shoulder at higher wavenumbers and the peaks at 71 and $137 \mathrm{~cm}^{-1}$, belonging to Form I, appear also in the spectra of the powders. 


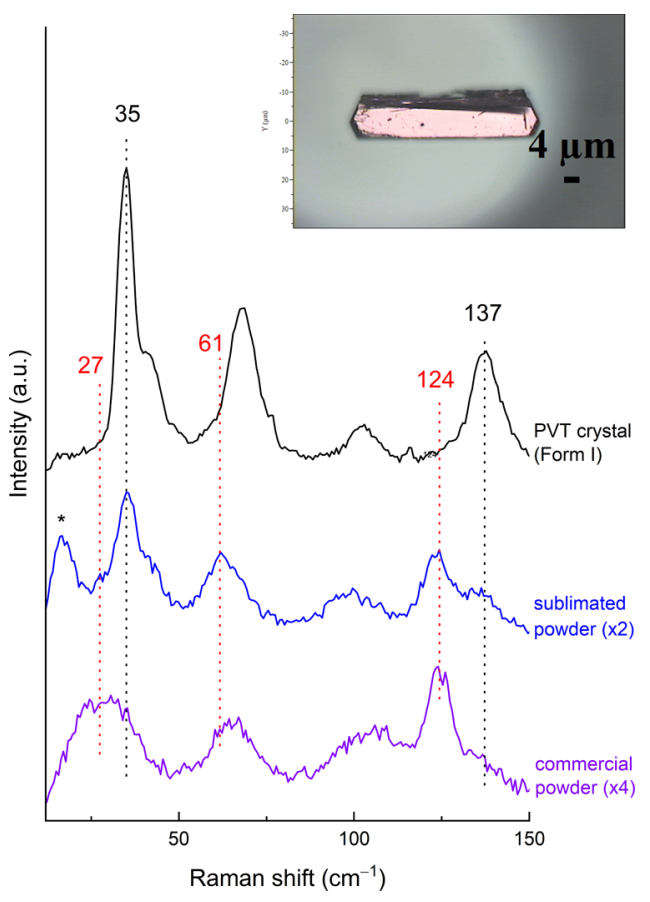

Figure 3. Raman spectra of a single crystal of 6,6'-dibromoindigo Form I obtained by the PVT method (inset), of a sublimated and of the commercial powders in the wavenumber range of the lattice phonon modes. $\lambda_{\text {exc }}=514.5 \mathrm{~nm}$. The peak with an asterisk is an experimental artifact.

Other features are either shifted and broadened or altogether new, like for instance the signal centered at around $27 \mathrm{~cm}^{-1}$ in the commercial product, which certainly originates from the convolution of more bands, or the bands observed at 61 and $124 \mathrm{~cm}^{-1}$, clearly identified in all of the powders. Thus, this is an unambiguous proof that both the commercial product and the sublimed material, in addition to the known Form I, contain a second structural modification that will be, in the following, identified as a new form, named from here on as Form II.

The evidence provided by the Raman spectra is supported also by the XRD analysis (see Supporting Information Figure S1-S3). The XRD pattern for Form II, apart from a small offset, appears to be very close to the one calculated from the triclinic $P \overline{1}$ SIP structures previously determined by a combined experimental and theoretical approach, namely that labeled A in ref 26. This prompted us to perform a Rietveld refinement on polycrystalline powders obtained by the sublimation method, starting from the SIP structure A, to get a reliable structure for the bulk sample.

The $\mathrm{Z}=1$ triclinic crystal structure thus determined for the new bulk form, $P \overline{1}$, with cell parameters (in $\AA$ and ${ }^{\circ}$ ) $a=$ 3.783(1), $b=5.826(2), c=15.32(1), \alpha=82.01(3), \beta=$ 85.32(4), $\gamma=86.32(5)$, and $V / Z=341.80(5) \AA^{3}$, is labeled Form II to distinguish it from the two closely related SIP structures A and B of ref 26. Crystal data and refinement details can be found in Table S1.

As shown in Figure 4, this new 6,6'-dibromoindigo bulk crystalline phase features a molecular arrangement quite different from that of Form I, even though it still displays the presence of strong intermolecular hydrogen and halogen bonds as well as $\pi-\pi$ stacking.

To fully probe spectroscopically the existence of a second crystal modification and in view of the analyses on surfacegrown crystallites (vide infra), it was necessary to identify and assign the bands of Form I in the diagnostic low-wavenumber region. This was done with the help of measurements and DFT-D3 vibrational calculations in polarized light, a combination which has proved to be effective for this task. ${ }^{16}$

Figure 5a reports the spectra of a thin platelet of $6,6^{\prime}$ dibromoindigo, aligned along the extinction direction and perpendicular to it. The excitation laser light was directed normal to the crystal face (100), as determined by XRD, and was linearly polarized in the same plane. Note that one of the crystal extinction directions necessarily coincides with the crystallographic $b$ axis of the monoclinic system.

(a)
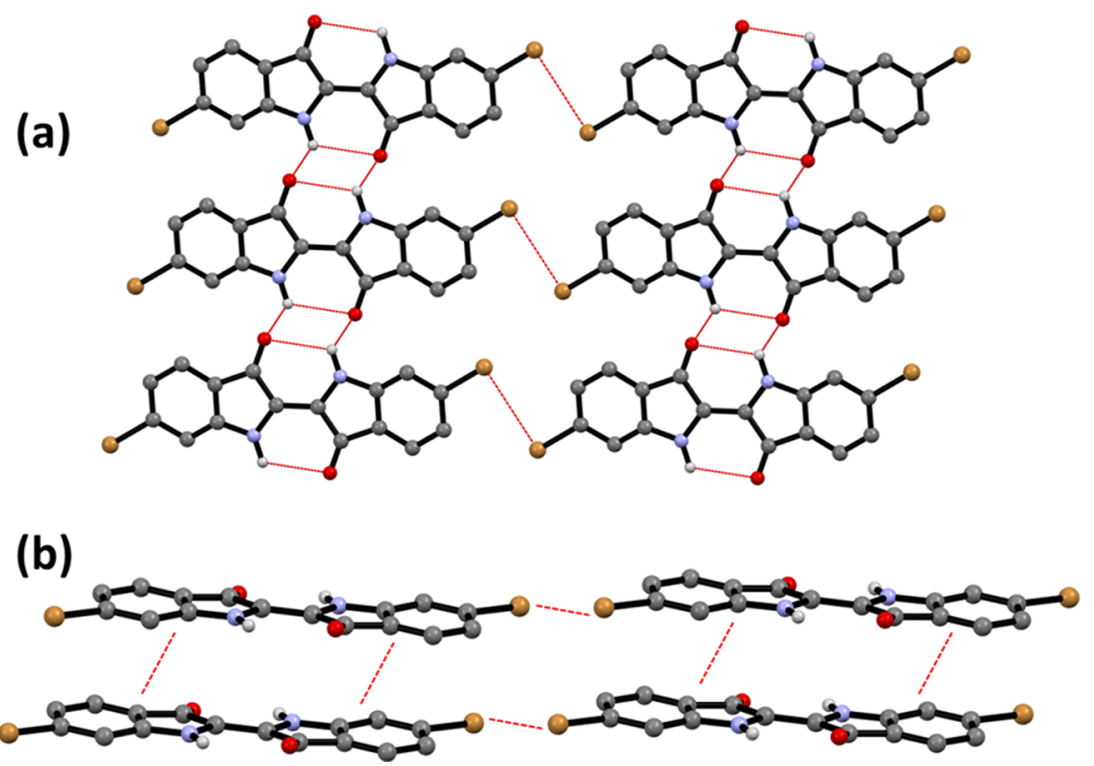

Figure 4. Intermolecular interactions detected in crystalline 6,6'-dibromoindigo Form II: (a) hydrogen bonds $\left[\mathrm{dN}_{\mathrm{NH}} \cdots \mathrm{O}_{\mathrm{CO}}\right.$, intermolecular $^{\prime}=$ $2.475(1) \AA$ and intramolecular $=3.201(9) \AA]$ and halogen bonds $\left[\mathrm{d}_{\mathrm{Br} \cdots \mathrm{Br}}=3.749(8) \AA\right.$, angle $\left.\mathrm{Br}-\mathrm{Br}-\mathrm{Br}=73.01(1)-106.99(1)^{\circ}\right]$; $(\mathrm{b}) \pi$-stacking $[3.797(3) \AA] . \mathrm{H}_{\mathrm{CH}}$ atoms are omitted for clarity. 

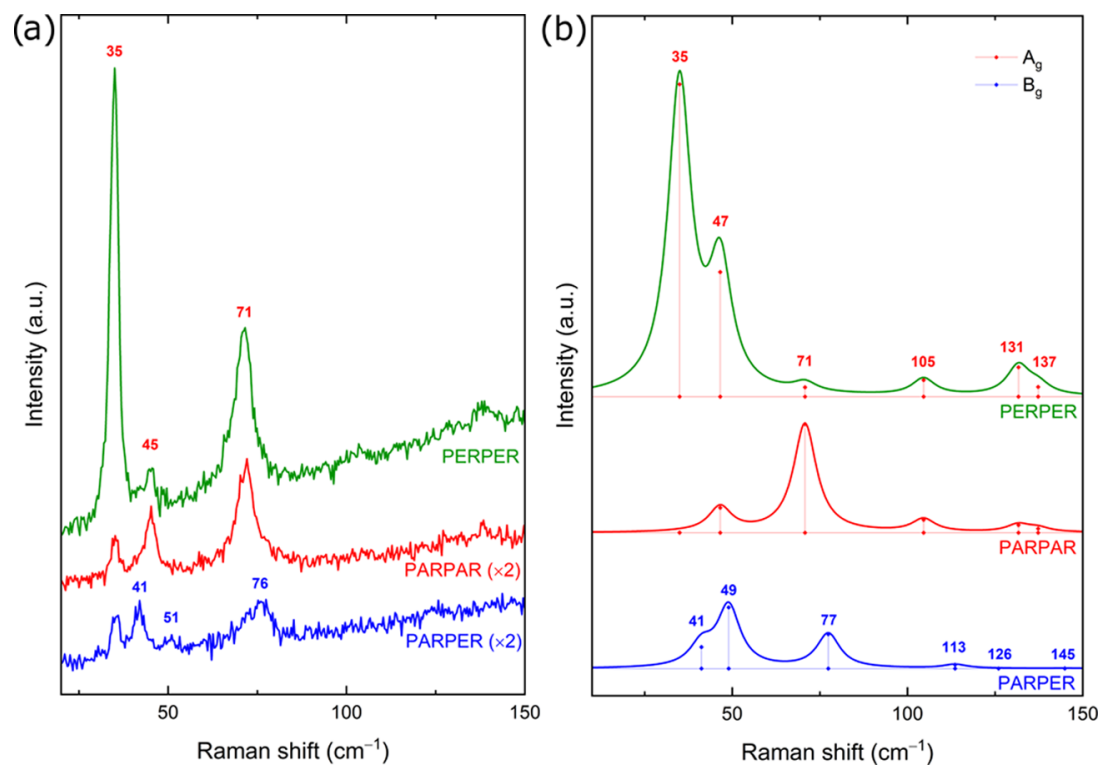

Figure 5. (a) Raman spectrum in polarized light of a single crystal of 6,6'-dibromoindigo Form I obtained by the PVT method, in the wavenumber range of the lattice phonon modes, with $\lambda_{\text {exc }}=647.1 \mathrm{~nm}$. (b) DFT-D3 Raman spectra for Form I, simulated in the experimental conditions. For the meaning of labels, see the text.

Table 1. Experimental (Form I and II) and Calculated (Form I, II, and SIPs) Wavenumbers of the Lowest Energy Modes of 6,6'-Dibromoindigo Crystals ${ }^{a}$

\begin{tabular}{|c|c|c|c|c|c|c|c|}
\hline \multicolumn{4}{|c|}{ Form I } & \multicolumn{2}{|c|}{ Form II } & \multicolumn{2}{|c|}{ SIPs and Form II } \\
\hline sym & $\exp \left(\mathrm{cm}^{-1}\right)$ & calcd $\left(\mathrm{cm}^{-1}\right)$ & rotation (\%) & sym & $\exp \left(\mathrm{cm}^{-1}\right)$ & calcd $\left(\mathrm{cm}^{-1}\right)$ & rotation (\%) \\
\hline$A_{g}$ & 35.0 & 35.0 & 100 & $A_{g}$ & 21.0 & $29.2,22.3,30.8$ & $99,100,99$ \\
\hline $\mathrm{B}_{\mathrm{g}}$ & 41.0 & 41.2 & 100 & $A_{g}$ & 39.0 & $40.9^{\prime} 27.8,37.3$ & $98,99,98$ \\
\hline$A_{g}$ & 45.0 & 46.6 & 99 & $A_{g}$ & 61.0 & $71.9,73.0,65.9$ & $61,64,73$ \\
\hline $\mathrm{B}_{\mathrm{g}}^{\mathrm{s}}$ & 51.0 & 49.0 & 99 & $A_{g}$ & 98.0 & $112.2,107.1,100.8$ & $24,21,18$ \\
\hline$A_{g}$ & 71.0 & 70.7 & 95 & $A_{g}$ & 124.0 & $133.6,130.5,127.2$ & $1,5,0$ \\
\hline $\mathrm{B}_{\mathrm{g}}$ & 76.0 & 77.4 & 87 & $A_{g}$ & & $145.6,144.5,138.4$ & $15,8,9$ \\
\hline$A_{g}$ & 103.0 & 104.6 & 4 & & & & \\
\hline $\mathrm{B}_{\mathrm{g}}$ & & 113.6 & 13 & & & & \\
\hline $\mathrm{B}_{\mathrm{g}}$ & 124 & 126.0 & 0 & & & & \\
\hline$A_{g}$ & & 131.6 & 0 & & & & \\
\hline$A_{g}$ & 137.0 & 137.3 & 0 & & & & \\
\hline $\mathrm{B}_{\mathrm{g}}$ & & 144.9 & 0 & & & & \\
\hline
\end{tabular}

${ }^{a}$ The "rotation" percentage is the total calculated squared rotational component $r_{x}^{2}+r_{y}^{2}+r_{z}^{2}$ around the three inertia axes $x, y$, and $z$, which for purely rotational "g" modes corresponds to the percentage of the lattice phonon character. The difference to 100 is the percentage of the intramolecular character of that mode. The three values reported for the calculations on Form II are obtained for the two different SIPs A and B of ref 26 and for the structure of Form II obtained in this work, in the given order.

The $k=0$ Raman-active modes of a $P 21 / c$ monoclinic system are of either $A_{g}$ or $B_{g}$ symmetry. If the electric vectors of the exciting and scattered electromagnetic waves both vibrate parallel or perpendicular to the $b$ axis, only $A_{g}$ modes are observed (PARPAR and PERPER labels in the figure); when the scattered wave is collected with polarization perpendicular to the excitation, only $B_{g}$ modes are instead detected (PARPER label in the figure). As confirmed by the calculations and similar to the parent system indigo, the lattice vibrational modes of 6,6'-dibromoindigo can be neatly separated into six lattice phonon modes, corresponding to pure translations and rotations of the rigid molecules, and intramolecular modes (rigid-body approximation). The former modes can be used to identify the lattice of Form I. The latter ones can be mapped onto the vibrations of the isolated molecule, bearing in mind that each molecular Raman-active mode splits into an $A_{g}+B_{g}$ Davydov's doublet in the crystal. In turn, 6,6'-dibromoindigo intramolecular vibrations strongly correlate with the indigo's modes, which we have previously analyzed and assigned. ${ }^{11}$ Polarized spectra have allowed us to solve the vibrational pattern corresponding to the six lattice phonon bands $\left(3 \mathrm{~A}_{\mathrm{g}}+3 \mathrm{~B}_{\mathrm{g}}\right)$ that characterize Form $\mathrm{I}$, with the calculations supporting the assignments, as shown in Figure $5 \mathrm{~b}$ and Table 1, where experimental and computed values are given. The table also reports the data on Form II, which will be discussed later on.

The only other bands detected and predicted for Form I over this interval are the strong ones at 103 and $137 \mathrm{~cm}^{-1}$ (DFT-D3 values at 104.6 and $137.3 \mathrm{~cm}^{-1}$, respectively). Lowwavenumber intramolecular modes of unexpectedly high intensities were also observed in indigo and found to be good markers for polymorph identification, as they are affected by the specific hydrogen bond pattern of the given structure. ${ }^{11}$ 
In conclusion, the vibrational fingerprint of Form I has been fully defined, and the investigation can move on to the results of the growths on the surface.

3.2. Characterization of Surface-Grown Samples. Figure 6 shows the typical image of the growth of
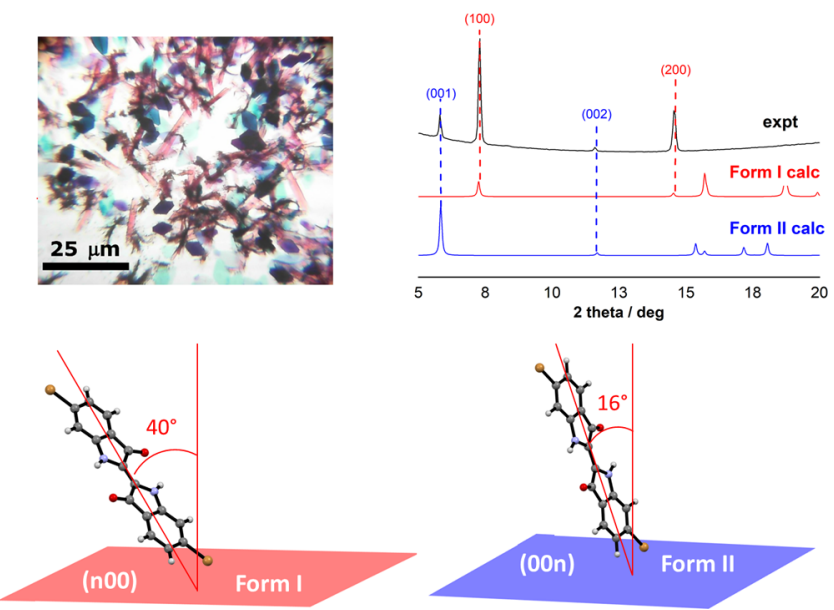

Figure 6. Top left: picture taken under polarized light of the crystalline materials grown on a glass surface. Top right: comparison between the XRD patterns recorded on a polycrystalline sample of $6,6^{\prime}$-dibromoindigo grown on a glass surface and the calculated ones for Form I and Form II. Bottom: orientation of molecules of 6,6'dibromoindigo Form I and Form II with respect to the appropriate Miller planes.

$6,6^{\prime}$ dibromoindigo on a glass surface collected in polarized light under an optical microscope, where it is possible to distinguish two different morphologies (plates and needles), associated with distinct pleochromic effects. In the figure, the $\mathrm{XRD}$ pattern collected on the ensemble of microcrystals is also reported. Unfortunately, the reduced size made it impossible for them to be detached from the substrate, and a separate single-crystal analysis for the two distinct morphologies could not be performed. However, two diffraction patterns can be identified in the growth. The first pattern corresponds to the (n00) series of $6,6^{\prime}$-dibromoindigo $P 21 / c$ Form I, which therefore has the plane $b c$ lying on the surface, in agreement with what was previously found in films grown by vapor deposition for this polymorph. ${ }^{17}$ In this geometry, the long molecular axis forms an angle of ca $40^{\circ}$ with the normal to the substrate. On the other hand, the second pattern appears to be very close to the peak series $(00 n)$ of the triclinic $P \overline{1}$ Form II determined in this work. This polymorph has the plane $a b$ lying on the surface, and the long molecular axis forms an angle of ca $16^{\circ}$ with the normal to the substrate.

It is worth recalling that various surface modifications have been found on different surfaces, characterized by slightly different values especially of the cell parameters $b$, along which the crystal hydrogen bond pattern develops, and $c$ of the triclinic structure. ${ }^{17,18}$

By subjecting the individual crystallites of the surface growth to Raman microscopy measurements, we were able to check whether the two structures identified by XRD could be associated with the different observed morphologies.

Figure 7a reports the Raman spectra collected on each of the two morphologies at low wavenumbers $\left(10-150 \mathrm{~cm}^{-1}\right)$. Namely, the blue trace is collected on a plate, which appears blue in the image under the polarizing microscope, and corresponds to that of Form I, despite the morphology being different from the PVT-grown sample. The red trace is collected on a needle oriented along its axis, which is found to coincide with an extinction direction. For this sample, three bands are observed in the range of the lattice phonons, at 21 , 39 , and $61 \mathrm{~cm}^{-1}$, accompanied by two more peaks at 98 and $124 \mathrm{~cm}^{-1}$, which have also been detected in the powder. A comparison with the spectrum of Form I also highlights the absence of the strong band at $137 \mathrm{~cm}^{-1}$, which is a relevant feature of Form I. This yields a lattice phonon and a lowenergy intramolecular mode of the Raman spectrum, which
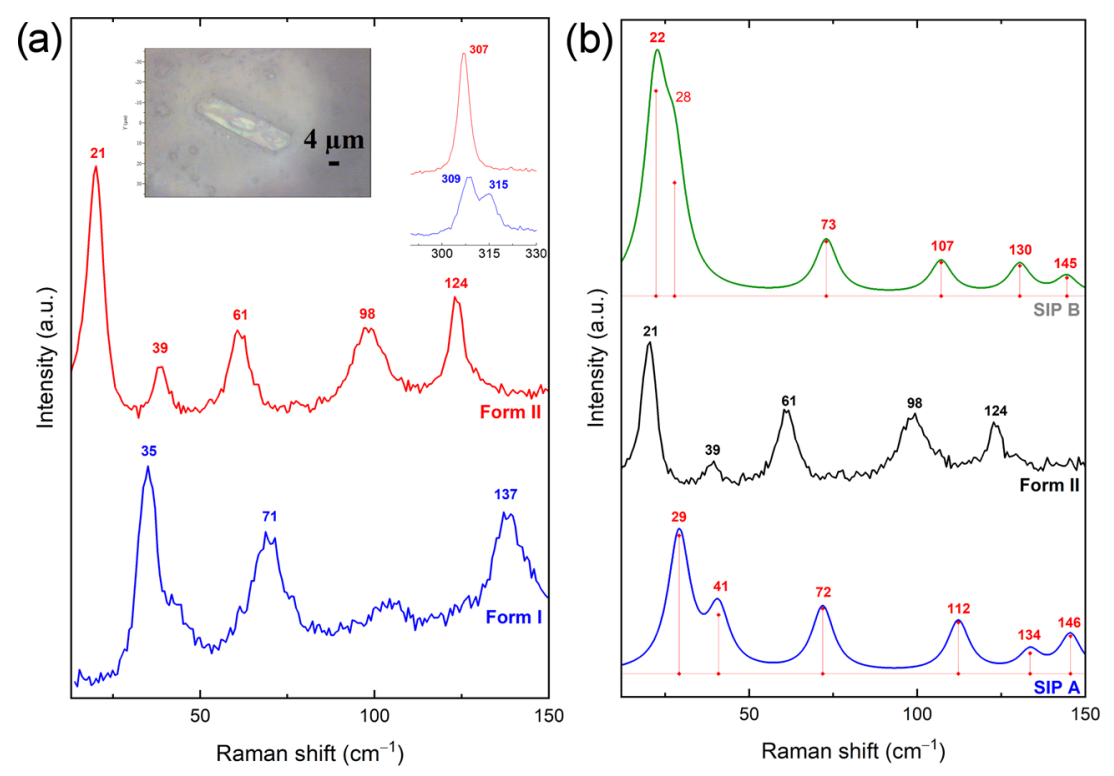

Figure 7. Raman spectra, in the wavenumber range of the lattice phonon modes for 6,6'-dibromoindigo crystallites grown after drop-casting: (a) the blue trace corresponds to the spectrum of specimens with a plate morphology and the red trace to the needlelike crystals (inset). (b) The spectrum of the needle is compared to the spectra simulated for the proposed SIP crystal structures, assuming in all cases $a b$ as the scattering face. $\lambda_{\text {exc }}=514.5 \mathrm{~nm}$. 
unequivocally identifies the Form II crystal structure, as confirmed by the comparison with the DFT D3-calculated wavenumbers reported in Table 1 for this modification.

Notably, the measurements in polarized light given in Figure S4 do not reveal any more bands over this range, displaying simply moderate relative intensity changes.

At higher energies, the spectra of Forms I and II also differ in an important detail (shown in the inset of Figure 6), which allows us to gather information about the symmetry of their respective unit cells. In Form I, the band at $309 \mathrm{~cm}^{-1}$ and its shoulder at $315 \mathrm{~cm}^{-1}$ can be solved by polarized measurements (Figure S5) into the components of a Davydov's doublet, with $\mathrm{A}_{\mathrm{g}}$ and $\mathrm{B}_{\mathrm{g}}$ symmetries, respectively. Note that the intermolecular coupling of the intramolecular modes is so weak in comparison to their energies that the resulting split is generally observed only for a few low-frequency vibrations, as in this case. Its detection, though, is worth noting here, as it represents the spectroscopic evidence of a centrosymmetric monoclinic lattice with two molecules per cell for Form I. On the contrary, the absence of the split for Form II is in agreement with the presence of just one molecule per unit cell.

To dispel any doubt about the chemical identity of the new Form II, its spectrum in the wavenumber range of 200-2000 $\mathrm{cm}^{-1}$ has been compared in Figure S6 with the spectra of Form I and of the powder. Except for some band splitting/shifting, all of these spectra are virtually identical, in agreement with the assumption that we are dealing with packing polymorphs of the same compound.

Altogether, the findings of both the $\mathrm{XRD}$ and Raman investigations suggest that Form II is a centrosymmetric structure with only one molecule per cell, which is compatible with a $P \overline{1}$ crystal symmetry. The key point is that Form II is present in both the samples obtained by drop-casting and the commercial and sublimed powders, in both cases concomitantly with Form I.

In Figure $7 \mathrm{~b}$, the experimental Raman spectrum of Form II is compared with the DFT D3-simulated spectra of two 6,6'dibromoindigo triclinic SIPs, while Table 1 gives the values of the corresponding peak wavenumbers. It is worth mentioning that the considered SIP structures are very similar to each other and indeed similar to the Form II of this work, as demonstrated by the $\mathrm{RMSD}_{N}$ values given in Table S3, which identify them as clearly clustering together.

Given the $P \overline{1}$ crystal symmetry of the triclinic system, the Raman experiment can detect only modes of $A_{g}$ symmetry, and with one molecule per unit cell, only three of these are expected to have the predominant character of lattice phonons in the rigid-body approximation. The eigenvector analysis given in Table 1 for these modes confirms this deduction. The spectra of Figure $7 \mathrm{~b}$ have been simulated for a crystal lying on the (001) face, excited by an electric vector vibrating on the same plane, so as to reproduce the orientation suggested by the experiments for the drop-cast samples.

The general agreement between experimental and simulated vibrational patterns and a direct comparison between the wavenumber values of Table 1 hint again at the similarities between Form II and the SIP structures. In weighing such similarities, some factors should be taken into consideration. First, the simulations have been performed on structures that are the best fits to experimental GIXRD data, with an expected margin of error larger than what is found when solving a structure from a single-crystal analysis; indeed, small structural variations may lead to large differences in the dynamics of the system, i.e., in its vibrational frequencies. Second, the Form II bulk structure and the SIPs reported in refs 17, 18 are not necessarily coincident, as the latter have been detected in thin films. Rather, they could be viewed as a cluster of structures related to a bulk form, possibly through distortion.

To further probe the relationship between the 6,6'dibromoindigo phases, a crystallite identified by its needle shape and spectrum as Form II was subjected to slow heating and was observed to transform into Form I at a temperature around $210^{\circ} \mathrm{C}$, as clearly demonstrated by the Raman data of Figure 8 . At around the same temperature, crystallites of Form

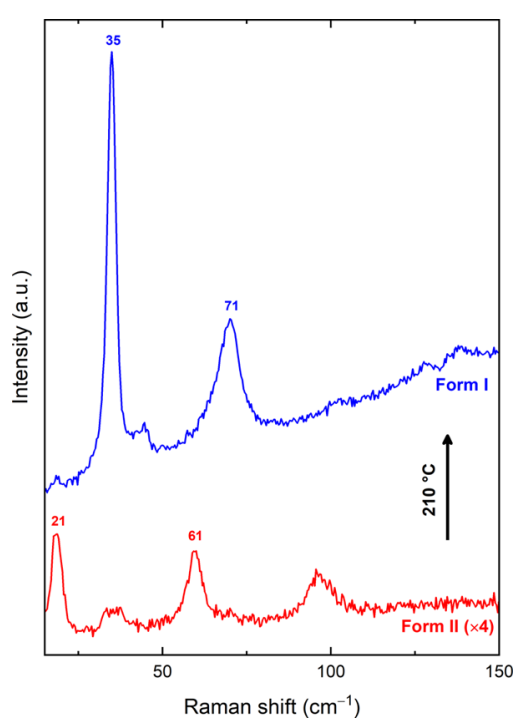

Figure 8. Raman spectra of a crystallite of 6,6'-dibromoindigo Form I subjected to heating: the spectrum transforms into that of Form II at $T=210{ }^{\circ} \mathrm{C} . \lambda_{\text {exc }}=514.5 \mathrm{~nm}$.

II appearing pinkish in polarized light underwent quite a sudden change of color, becoming blue as the plates of Form I. The transition seems to be irreversible, as the spectrum (and the color) of Form II cannot be recovered on cooling. This result, indeed, is in very good agreement with what was reported in ref 17, where the irreversible temperature-driven phase transition that transforms the surface-mediated structure into the bulk takes place at nearly the same temperature.

Some comments must be made on the different colors that Form I and Form II display in polarized light. Tyrian purple pleochromism, ${ }^{2}$ i.e., its color variation with light polarization, was attributed to the dependence of the absorption coefficient on whether the electric field $\mathrm{E}$ of the radiation vibrates on the molecular plane parallel or perpendicular to the direction of the central $\mathrm{C}=\mathrm{C}$ bond of the chromophore group. In the first case, the absorption maximum is centered at $640 \mathrm{~nm}$ and in the second at $540 \mathrm{~nm}^{2}$ In the geometry of our experimental setup, E vibrates on a focal plane parallel to the contact plane of the crystallites on the glass substrate, which is (100) and (001) for Form I and II, respectively. The smaller the angle formed by the long molecular axis with the normal to such a plane, the lower the absorption of the component parallel to the double bond, in the limit that in a "standing-up" molecular arrangement at the interface, only the $540 \mathrm{~nm}$ component should be present, imparting a red-violet color to the specimen. The difference in color observed between Form I plates and Form II needles should therefore be traced back to this effect: in Form I, with an angle of $\approx 40^{\circ}$, polarized light both parallel 
and perpendicular to the double bond can be absorbed; Form II instead, with an angle of $\approx 16^{\circ}$, approaches the condition of a standing-up geometry, a characteristic shared with the surfaceinduced structures labeled $\mathrm{A}$ and $\mathrm{B}$, which display angles of 21 and $29^{\circ}$, respectively. ${ }^{26}$

\section{CONCLUSIONS}

In the previously investigated hydrogen-bonded vat dyes indigo and thioindigo, it was found that in the growth at solid interfaces and film formation the substrate acts as a selector toward one of the known bulk forms of the system. ${ }^{11,16}$ Notwithstanding the strong structural similarities with its parent molecule, 6,6'-dibromoindigo appears to behave in a peculiar way. On one hand, it has been reported to display genuine surface-induced structures. In fact, depending on the nature and the treatment undergone by the surface, ${ }^{18}$ slightly different SIPs have been detected. On the other hand, the findings of this work suggest that these structures can be traced back to a bulk structure identified both structurally and spectroscopically through the synergic use of X-ray data, Raman analysis, and DFT spectra simulations.

The result reinforces the conviction that in many instances the structures present in thin films belong to phases already existing as energy minima in the polymorph landscape of the compound, independent of the substrate itself. In turn, it also stresses the importance of crystal structure prediction procedures in assisting the structure solving in these systems.

\section{ASSOCIATED CONTENT}

\section{SI Supporting Information}

The Supporting Information is available free of charge at https://pubs.acs.org/doi/10.1021/acs.jpcc.0c05186.

XRD analysis; crystal data, and refinement details of crystalline 6,6'-dibromoindigo Form I and Form II; polarized Raman spectrum of Form I in the lattice phonon region; polarized measurements of Davydov's doublet for Form I; intramolecular spectral comparison of the two forms with the powder; and $\mathrm{RMSD}_{N}$ values (PDF)

\section{AUTHOR INFORMATION}

\section{Corresponding Authors}

Tommaso Salzillo - Department of Molecular Nanoscience and Organic Materials, Institut de Ciencia de Materials de Barcelona (ICMAB-CSIC) and (CIBER-BBN), 08193 Bellaterra, Spain; (1) orcid.org/0000-0002-9737-2809;

Email: tommaso.salzillo@unibo.it

Elisabetta Venuti - Department of Industrial Chemistry "Toso Montanari" and INSTM-UdR Bologna, University of Bologna, 40136 Bologna, Italy; 이이이.org/0000-0003-3493-7953; Email: elisabetta.venuti@unibo.it

\section{Authors}

Lorenzo Pandolfi - Department of Industrial Chemistry "Toso Montanari" and INSTM-UdR Bologna, University of Bologna, 40136 Bologna, Italy

Arianna Rivalta - Department of Industrial Chemistry "Toso Montanari" and INSTM-UdR Bologna, University of Bologna, 40136 Bologna, Italy; (i) orcid.org/0000-0002-0528-4044

Andrea Giunchi - Department of Industrial Chemistry "Toso Montanari" and INSTM-UdR Bologna, University of Bologna, 40136 Bologna, Italy
Simone D’Agostino - Department of Chemistry "G. Ciamician”, University of Bologna, 40126 Bologna, Italy; (1) orcid.org/0000-0003-3065-5860

Raffaele G. Della Valle - Department of Industrial Chemistry "Toso Montanari" and INSTM-UdR Bologna, University of Bologna, 40136 Bologna, Italy; 이이.org/0000-00020502-5894

Aldo Brillante - Department of Industrial Chemistry "Toso Montanari" and INSTM-UdR Bologna, University of Bologna, 40136 Bologna, Italy

Complete contact information is available at:

https://pubs.acs.org/10.1021/acs.jpcc.0c05186

\section{Author Contributions}

The manuscript was written through contributions of all authors. All authors have given approval to the final version of the manuscript.

Notes

The authors declare no competing financial interest.

\section{ACKNOWLEDGMENTS}

We thank CINECA Supercomputing Center for providing computer time through the ISCRA scheme (project CSCSCDFT). T.S. acknowledges the H2020-MSCA-COFUND2014 Programme (P-SPHERE, Grant agreement 665919). The authors thank Prof. Roland Resel for the fruitful discussions. This work has been funded by the PRIN project "From natural to artificial light-harvesting systems: unveiling fundamental processes toward a bio-inspired materials design" (HARVEST) protocol 201795 SBA3.

\section{REFERENCES}

(1) Cooksey, C. J. Tyrian Purple: 6,6'-Dibromoindigo and Related Compounds. Molecules 2001, 6, 736-769.

(2) Süsse, P.; Krampe, C. 6,6'-Dibromo-Indigo, a Main Component of Tyrian Purple. Naturwissenschaften 1979, 66, 110.

(3) Larsen, S.; Wätjen, F. The Crystal and Molecular Structures of Tyrian Purple (6,6'-Dibromoindigotin) and 2,2'-Dimethoxyindigotin. Acta Chem. Scand., Ser. A 1980, 34, 171-176.

(4) Głowacki, E. D.; Voss, G.; Leonat, L.; Irimia-Vladu, M.; Bauer, S.; Sariciftci, N. S. Indigo and Tyrian Purple - From Ancient Natural Dyes to Modern Organic Semiconductors. Isr. J. Chem. 2012, 52, 540-551.

(5) Głowacki, E. D.; Leonat, L.; Voss, G.; Bodea, M.-A.; Bozkurt, Z.; Ramil, A. M.; Irimia-Vladu, M.; Bauer, S.; Sariciftci, N. S. Ambipolar Organic Field Effect Transistors and Inverters with the Natural Material Tyrian Purple. AIP Adv. 2011, 1, No. 042132.

(6) Shityakov, S.; Roewer, N.; Förster, C.; Broscheit, J. A. In Silico Modeling of Indigo and Tyrian Purple Single-Electron NanoTransistors Using Density Functional Theory Approach. Nanoscale Res. Lett. 2017, 12, 439.

(7) Irimia-Vladu, M.; Kanbur, Y.; Camaioni, F.; Coppola, M. E.; Yumusak, C.; Irimia, C. V.; Vlad, A.; Operamolla, A.; Farinola, G. M.; Suranna, G. P.; González-Benitez, N.; et al. Stability of Selected Hydrogen Bonded Semiconductors in Organic Electronic Devices. Chem. Mater. 2019, 31, 6315-6346.

(8) Anokhin, D. V.; Leshanskaya, L. I.; Piryazev, A. A.; Susarova, D. K.; Dremova, N. N.; Shcheglov, E. V.; Ivanov, D. A.; Razumov, V. F.; Troshin, P. A. Towards Understanding the Behavior of Indigo Thin Films in Organic Field-Effect Transistors: A Template Effect of the Aliphatic Hydrocarbon Dielectric on the Crystal Structure and Electrical Performance of the Semiconductor. Chem. Commun. 2014, 50, 7639-7641. 
(9) Scherwitzl, B.; Resel, R.; Winkler, A. Film Growth, Adsorption and Desorption Kinetics of Indigo on SiO2. J. Chem. Phys. 2014, 140, No. 184705.

(10) Irimia-Vladu, M.; Gåowacki, E. D.; Troshin, P. A.; Schwabegger, G.; Leonat, L.; Susarova, D. K.; Krystal, O.; Ullah, M.; Kanbur, Y.; Bodea, M. A.; et al. Indigo-A Natural Pigment for High Performance Ambipolar Organic Field Effect Transistors and Circuits. Adv. Mater. 2012, 24, 375-380.

(11) Salzillo, T.; D’Agostino, S.; Rivalta, A.; Giunchi, A.; Brillante, A.; Della Valle, R. G.; Bedoya-Martínez, N.; Zojer, E.; Grepioni, F.; Venuti, E. Structural, Spectroscopic, and Computational Characterization of the Concomitant Polymorphs of the Natural Semiconductor Indigo. J. Phys. Chem. C 2018, 122, 18422-18431.

(12) Rivalta, A. A Multi-Methodological Approach for the Study of Polymorphism in Organic Pigments and Pharmaceuticals; University of Bologna, 2019.

(13) Von Eller-Pandraud, H. Sur Le Polymorphisme de l'indigo. Bull. Soc. Chim. Fr. 1955, 1, 316-317.

(14) Von Eller, H. Structure de Colorants Indigoides. III-Structure Cristalline de l'indigo. Bull. Soc. Chim. Fr. 1955, 1433-1438.

(15) Kettner, F.; Hüter, L.; Schäfer, J.; Röder, K.; Purgahn, U.; Krautscheid, H. Selective Crystallization of Indigo B by a Modified Sublimation Method and Its Redetermined Structure. Acta Crystallogr., Sect. E: Struct. Rep. Online 2011, E67, o2867.

(16) Rivalta, A.; Giunchi, A.; Pandolfi, L.; Salzillo, T.; D’agostino, S.; Werzer, O.; Schrode, B.; Demitri, N.; Mas-Torrent, M.; Brillante, A.; et al. Crystal Alignment of Surface Stabilized Polymorph in Thioindigo Films. Dyes Pigm. 2020, 172, No. 107847.

(17) Truger, M.; Roscioni, O. M.; Röthel, C.; Kriegner, D.; Simbrunner, C.; Ahmed, R.; Glowacki, E. D.; Simbrunner, J.; Salzmann, I.; Coclite, A. M.; et al. Surface-Induced Phase of Tyrian Purple (6,6'-Dibromoindigo): Thin Film Formation and Stability. Cryst. Growth Des. 2016, 16, 3647-3655.

(18) Truger, M.; Jones, A. O. F.; Coclite, A. M.; Pachmajer, S.; Kriegner, D.; Röthel, C.; Simbrunner, J.; Salzmann, I.; Resel, R. Crystallization of Tyrian Purple (6,6'-Dibromoindigo) Thin Films: The Impact of Substrate Surface Modifications. J. Cryst. Growth 2016, 447, 73-79.

(19) Salzillo, T.; Rivalta, A.; Castagnetti, N.; D’Agostino, S.; Masino, M.; Grepioni, F.; Venuti, E.; Brillante, A.; Girlando, A. Spectroscopic Identification of Quinacridone Polymorphs for Organic Electronics. CrystEngComm 2019, 21, 3702-3708.

(20) Bedoya-Martínez, N.; Schrode, B.; Jones, A. O. F.; Salzillo, T.; Ruzié, C.; Demitri, N.; Geerts, Y. H.; Venuti, E.; Della Valle, R. G.; Zojer, E.; et al. DFT-Assisted Polymorph Identification from Lattice Raman Fingerprinting. J. Phys. Chem. Lett. 2017, 8, 3690-3695.

(21) Salzillo, T.; Giunchi, A.; Masino, M.; Bedoya-Martı'nez, N.; Della Valle, R. G.; Brillante, A.; Girlando, A.; Venuti, E. An Alternative Strategy to Polymorph Recognition at Work: The Emblematic Case of Coronene. Cryst. Growth Des. 2018, 18, 4869-4873.

(22) Sheldrick, G. M. SHELX-97, Program for Crystal Structure Determination; University of Gottingen: Gottingen, Germany, 1997.

(23) Macrae, C. F.; Bruno, I. J.; Chisholm, J. A.; Edgington, P. R.; McCabe, P.; Pidcock, E.; Rodriguez-Monge, L.; Taylor, R.; van de Streek, J.; Wood, P. A. Mercury CSD 2.0-New Features for the Visualization and Investigation of Crystal Structures. J. Appl. Crystallogr. 2008, 41, 466-470.

(24) Macrae, C. F.; Sovago, I.; Cottrell, S. J.; Galek, P. T. A.; McCabe, P.; Pidcock, E.; Platings, M.; Shields, G. P.; Stevens, J. S.; Towler, M.; et al. Mercury 4.0: From Visualization to Analysis, Design and Prediction. J. Appl. Crystallogr. 2020, 53, 226-235.

(25) Cohelo, A. TOPAS-Academic, Coelho Software: Brisbane; Coelho Software: Brisbane, Australia, 2007.

(26) Resel, R. Private Communication: Lattice Parameters (a, b, c, $\alpha$, $\beta, \gamma$, in $\AA$ and o) Determined for Two Different SIP Samples (Labeled $\mathrm{A}$ and $\mathrm{B}$ ) Grown on $\mathrm{Si} / \mathrm{SiO} 2$ Substrates Were $(3.86,5.83,14.86,81.8$, $85.8,87.1)$ and $(3.84,6.00,14.67,86.0,87.0,87.0)$, Respectiv.

(27) Kresse, G.; Hafner, J. Ab Initio Molecular Dynamics for Liquid Metals. Phys. Rev. B 1993, 47, 558-561.
(28) Kresse, G.; Hafner, J. Ab Initio Molecular-Dynamics Simulation of the Liquid-Metal-Amorphous-Semiconductor Transition in Germanium. Phys. Rev. B 1994, 49, 14251-14269.

(29) Kresse, G.; Furthmüller, J. Efficiency of Ab-Initio Total Energy Calculations for Metals and Semiconductors Using a Plane-Wave Basis Set. Comput. Mater. Sci. 1996, 6, 15-50.

(30) Kresse, G.; Furthmüller, J. Efficient Iterative Schemes for Ab Initio Total-Energy Calculations Using a Plane-Wave Basis Set. Phys. Rev. B 1996, 54, 11169-11186.

(31) Perdew, J. P.; Burke, K.; Ernzerhof, M. Generalized Gradient Approximation Made Simple. Phys. Rev. Lett. 1996, 77, 3865-3868.

(32) Blöchl, P. E. Projector Augmented-Wave Method. Phys. Rev. B 1994, 50, 17953-17979.

(33) Kresse, G.; Joubert, D. From Ultrasoft Pseudopotentials to the Projector Augmented-Wave Method. Phys. Rev. B 1999, 59, 17581775.

(34) Grimme, S.; Ehrlich, S.; Goerigk, L. Effect of the Damping Function in Dispersion Corrected Density Functional Theory. J. Comput. Chem. 2011, 32, 1456-1465.

(35) Bučko, T.; Hafner, J.; Ángyán, J. G. Geometry Optimization of Periodic Systems Using Internal Coordinates. J. Chem. Phys. 2005, 122, No. 124508.

(36) Chisholm, J. A.; Motherwell, S. COMPACK: A Program for Identifying Crystal Structure Similarity Using Distances. J. Appl. Crystallogr. 2005, 38, 228-231.

(37) Togo, A.; Tanaka, I. First Principles Phonon Calculations in Materials Science. Scr. Mater. 2015, 108, 1-5.

(38) Fornari, A.; Stauffer, S. Vasp raman.Py; GitHub, 2013.

(39) Michalska, D.; Wysokiński, R. The Prediction of Raman Spectra of Platinum(II) Anticancer Drugs by Density Functional Theory. Chem. Phys. Lett. 2005, 403, 211-217. 